\title{
The non-operant nature of the bar-press escape response
}

\author{
ROBERT C. BOLLES AND DANIEL B. McGILLIS ${ }^{2}$, \\ DEPARTMENT OF PSYCHOLOGY, UNIVERSITY OF \\ WASHINGTON, Seattle, Wash. 98105
}

Fifty-six rats were trained to press a bar to terminate shock. Different groups were trained with different force requirements and different response feedback conditions. Under most conditions the modal response latency became very short, $0.1 \mathrm{sec}$ or less, which indicates that at least much of the time the response should not be considered an operant.

The purpose of this paper is to propose two heresies. One is that the bar-press response is not necessarily a purely arbitrary selection of a convenient piece of emitted behavior; under some rather commonly encountered conditions it is not an operant. The second is that the rate of bar pressing is not necessarily increased by making shock termination contingent upon its occurrence; under some conditions a high rate of bar pressing occurs which cannot be attributed to reinforcement. Our case is based upon the very short latency of the bar press escape response.

Method. The Ss were 56 naive female rats of Wistar origin, approximately four months old. They were trained in a Foringer box, Model $1102 \mathrm{M}$ with a modified bar. The telegraph key switch that is normally operated by the Foringer bar was replaced by a rigid metal rod so that the bar itself could not be perceptibly moved. An E \& M model F-1000 electromyographic detector was attached to this rod to detect the force $S$ put on the bar. The detector signal was amplified so that, for different groups, a force of $5,10,20$ or $40 \mathrm{~g}$ on the bar would operate a flip-flop in the programming apparatus. The program included a trigger circuit so that $S$ had to release the bar and press it again with the specified force to produce a defined response. Shock was approximately $1 \mathrm{~mA}$ dc presented with a Foringer shock scrambler on a variable schedule $($ mean $=46 \mathrm{sec})$.

The Ss were trained with one of the four force requirements, and either with or without response feedback in a 4 by 2 design. Feedback was provided by the click of a relay mounted in the box. Because of the use of the rigid bar and transistorized equipment an intertrial response in the absence of a programmed click (the No-feedback condition) yielded no response feedback other than whatever kinesthetic factors may have been intrinsic in the response. A response in the presence of shock of course terminated it. The response was not shaped; $S$ was merely put in the box and the shock programmer turned on. Ss were trained a single session of 50 trials and escape latencies were recorded to $.01 \mathrm{sec}$.

Results. Nearly all Ss learned the response in the sense of showing progressively decreasing latencies. All groups showed rapid acquisition and a high level of performance. For different groups the median latency over the first block of 10 trials ranged from approximately 4 to $12 \mathrm{sec}$, while the median latency over Trials 41-50 ranged from .07 to $1.3 \mathrm{sec}$. There was some suggestion of faster acquisition and faster responding among the feedback groups and among the lighter force groups, but with only seven in a group none of these effects approached significance. The real function of the different groups was not to demonstrate the effects of the experimental variables but rather to show the generality of the principal findings. We are not interested here in the differences among groups but in their similarity.

Our chief interest is in the overall distribution of response latencies during the last 10 trials, which is shown in Fig. 1. Notice that the class intervals are given on a geometric scale. If the distribution was plotted on a linear scale it would be much more skewed.

The overall mean of the distribution shown in Fig. 1 is $1.5 \mathrm{sec}$, a value consistent with those previously reported (e.g., D'Amato, Keller, \& Biederman, 1965; Dinsmoor \& Hughes, 1956), and consistent with the common assumption that the bar press is an operant response. But on $50 \%$ of the trials the latency was. $14 \mathrm{sec}$ or less, and the most common or modal recorded value was $.05 \mathrm{sec}$. The occurrence of response-terninated shocks of 40 and 50 msec duration was confirmed with an oscilloscope. We submit that in these instances of $.05 \mathrm{sec}$ latencies the response. simply cannot be an operant.

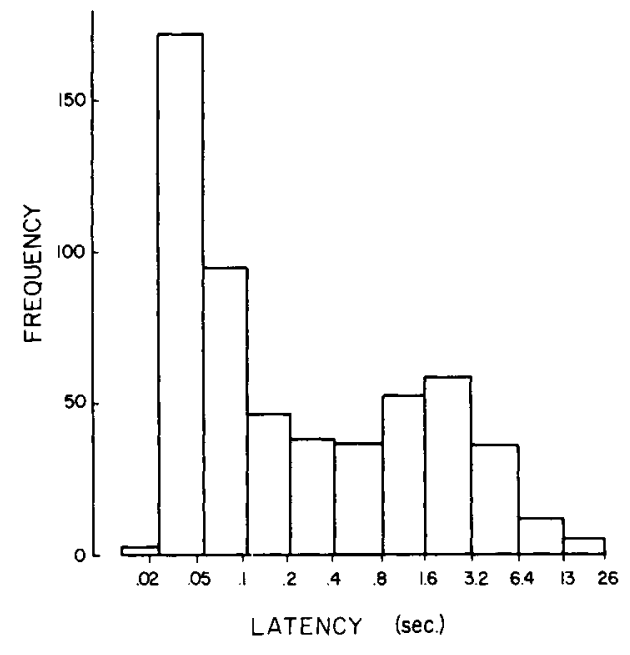

Fig. 1. Frequency distribution of escape latencies $(N=560)$.

Discussion. Other experimenters (e.g., Bolles \& Warren, 1965; D'Amato et al, 1965; Migler, 1963) have either stated or implied that bar press escape latencies can be extremely short, but just how short these latencies are, and what this phenomenon implies for aversive conditioning have not been explored. The left-hand peak in Fig. 1 represents a class of responses with latencies too short for them to define an operant. We know from observing Ss that such responses occur when $S$ assumes a static posture holding onto the bar. We assume that when shock comes on there is either a reflexive lurch or perhaps a direct current-induced muscular contraction that results in the bar being depressed.

We know too that there is at least some sense in which this behavior is learned, because in earlier blocks of trials the left-hand peak in Fig. 1 is much less pronounced while the right-hand peak is correspondingly larger and farther to the right. We assume that what $S$ learns is to acquire the static body posture (freezing) that permits the reflexive short-latency responding to occur. Some Ss acquire this mode of responding with 10 trials or less, while a few apparently never acquire it. Acquisition of fast responding was found here to be facilitated by minimizing the force required on the bar. and perhaps it can be reduced or eliminated by increasing the amount of movement required on the bar (e.g., Dinsmoor \& Hughes, 1956), but that is conjectural.

Superficially, and at a purely procedural level, this acquisition would appear to be reinforced by the shock-termination contingency. But we believe that this is not the case, primarily because we have consistently obtained precisely the same kind of latency distributions using short shocks, i.e., .1-sec pulses presented once per sec.

We suppose that freezing is very high in the rat's response repertoire when it has been shocked or otherwise frightened. Put in a small, confining box the rat will freeze during most of the intershock interval. Freezing can only persist during the presentation of shock if the rat freezes while holding onto the bar, and, more specifically, while holding onto the bar in such a way that short-latency reflexive responses can occur. In short, we propose that the short-latency mode of responding is shaped up by the differential punishment of all other behavior, rather than by the escape contingency itself. If this is the case, it provides further support for the argument that in these instances the bar press is not an operant. We suggest that freezing on the bar is an operant, and that it is under control of the escape contingency, but that the resulting short-latency responses themselves are not.

Finally, we propose that it is the rapid acquisition and great persistence of this highly effective mode of responding (effective in the sense that it minimizes shock) that interfere with the acquisition of bar-press avoidance behavior and makes the latter so difficult to establish.

\section{REFERENCES}

BOLLES, R. C., \& WARREN. J. A., Jr. Effects of delayed UCS temination on classical avoidance learning of the bar-press response. Psychol. $R c^{\prime} p$. $1965,17,687-690$. 
D'AMATO, M. R., KELLER, D., \& BIEDERMAN, G. Discriminated avoidance learning as a function of parameters of discontinuous shock. $J$. exp. Psychol., 1965, 70, 543-548.

DINSMOOR, J. A., \& HUGHES, L. H. Training rats to press a bar to turn off shock. J. comp. physiol Psychol., 1956, 49, 235-238.

MIGLER, B. Bar holding during escape conditioning. J. exp. Anal. Behav.,
$1963,6,65-72$.

\section{NOTES}

1. Supported by Research Grant GB-5694 from the National Science Foundation.

2. Now at Duke University, Durham, North Carolina. 